\title{
Electrical Modeling of the Influence of Medium Conductivity on Electroporation
}

\author{
Antoni Ivorra $*, 1$, Julien Villemejane ${ }^{2,3,4}$ and Lluis M. Mir ${ }^{2,3}$ \\ ${ }^{1}$ Dept. of Information and Communication Technologies, Universitat Pompeu Fabra, Barcelona, \\ Spain \\ ${ }^{2}$ CNRS, UMR 8203, Institut Gustave-Roussy, Villejuif, France \\ ${ }^{3}$ Univ Paris-Sud, UMR 8203 \\ ${ }^{4}$ CNRS, SATIE, Institut d'Alembert, ENS Cachan, Cachan, France \\ ${ }^{*}$ Correspondig author \\ E-mail address: antoni.ivorra@gmail.com
}

\begin{abstract}
Electroporation is the phenomenon in which cell membrane permeability is increased by exposing the cell to short high electric field pulses. Experimental data shows that the amount of permeabilization depends on the conductivity of the extracellular medium. If medium conductivity decreases then it is necessary to deliver a pulse of larger field amplitude in order to achieve the same effect. Models that do not take into account the permeabilization effect on the membrane conductivity cannot reproduce qualitatively the experimental observations. Here we employ an exponential function for describing the strong dependence of membrane conductivity on transmembrane potential. Combining that model with numerical methods we demonstrate that the dependence on medium conductivity can be explained as being the result of increased membrane conductance due to electroporation. As experimentally observed, extracellular conductivities of about $1 \mathrm{~S} / \mathrm{m}$ and $0.1 \mathrm{~S} / \mathrm{m}$ yield very similar results, however, for lower conductivities $(<0.01 \mathrm{~S} / \mathrm{m}$ ) the model predicts that significantly higher field magnitudes will be required to achieve the same amount of permeabilization.
\end{abstract}

\section{Introduction}

Electroporation, or electropermeabilization, is the phenomenon in which cell membrane permeability to ions and macromolecules is increased by exposing the cell to short (microseconds to milliseconds) high electric field pulses. The permeabilization can be either temporary (reversible electroporation) or permanent (irreversible electroporation) as a function of the electrical field magnitude and duration, pulse repetition frequency and number of the pulses. In vitro, reversible electroporation is now commonly used for gene transfection of cells in culture ${ }^{1}$ whereas irreversible electroporation is considered as a potential viable method for cold sterilization of liquid media ${ }^{2,3}$. In addition, reversible electroporation is also used in living tissues for in vivo gene therapy ${ }^{4}$ and to enhance the penetration of anti-cancer drugs into undesirable cells (electrochemotherapy, ECT) ${ }^{5}$. Recently, irreversible electroporation (IRE) has also found a use in tissues as a minimally invasive surgical procedure to ablate undesirable tissue without the use of adjuvant drugs ${ }^{6}$.

Multiple in vitro experimental studies demonstrate that the extracellular medium conductivity $\left(\sigma_{e}\right)$ has an influence on the electroporation phenomenon ${ }^{1,7-12}$. In five out of seven of these cited studies, it was observed that electroporation efficiency decreases when medium 
conductivity decreases but in the other two the opposite effect was observed, that is, efficiency decreased when medium conductivity increased ${ }^{7}$, 9 . In those two singular cases the pulse generator consisted of a capacitance charged to a high voltage that was allowed to discharge through the medium in which cells where contained. That is the oldest method used to generate electroporation pulses ${ }^{13}$ and it has a well identified drawback when compared to modern square pulse generators: in these capacitance-discharge generators the applied pulse length depends on medium conductivity. And such dependence would explain the exceptional observation for those two cases: as medium conductivity increases the pulse length decreases and as a consequence the intensity of the electroporation phenomenon diminishes ${ }^{14}$. Observations in all the other studies can be summarized as follows: 1) electroporation efficiency decreases as $\sigma_{e}$ decreases, 2) decrease rate in efficiency as a function of $\sigma_{e}$ is higher for lower $\sigma_{e}$ values and 3) electroporation efficiency sensitivity to $\sigma_{e}$ varies significantly between experiments and depending on the way permeabilization is assessed. Here it is convenient to note that in the cited studies the depletion of ions from the medium was osmotically counterbalanced with neutral compounds such as sucrose. Otherwise, it could be reasonably argued that it is the osmolarity, and not the electrical conductivity, the main factor that influences electroporation. ${ }^{15}$

If we define a "critical" electric field magnitude, $E_{c}$, at which the electroporation effect is quantitatively the same for different extracellular conductivities, then it is possible to plot data from some of above cited studies ${ }^{1,10,11}$ so that the above observations are illustrated in a comprehensible way (Fig. 1). The other two studies ${ }^{8,12}$ show that electroporation efficiency diminishes as $\sigma_{e}$ decreases but do not provide data for representing $E_{c}$ at different extracellular conductivities.

The cases B and D on Fig. 1 are particularly illustrative. Both cases correspond to the same study and followed the same experimental procedures apart from the method employed for assessing electroporation efficiency. In $\mathrm{D}$ cell reversible permeabilization was evaluated by quantifying cell survival rate after electroporation in a medium containing bleomycin, which is a powerful cytotoxic drug barely able to penetrate the cells under normal conditions. In B direct cell death (necrosis) caused by irreversible permeabilization was quantified. As it can be observed in $\mathrm{D}$, which is the case that requires a lower level of permeabilization, the permeabilization sensitivity to $\sigma_{e}$ is almost insignificant whereas in $\mathrm{B}$, which requires a higher level of permeabilization, the required field magnitude increases as $\sigma_{e}$ decreases. Moreover, such field sensitivity to $\sigma_{e}$ seems to become larger as $\sigma_{e}$ decreases. The motivation of the present study was to analyze whether it was possible to justify such patterns in electrical terms. 


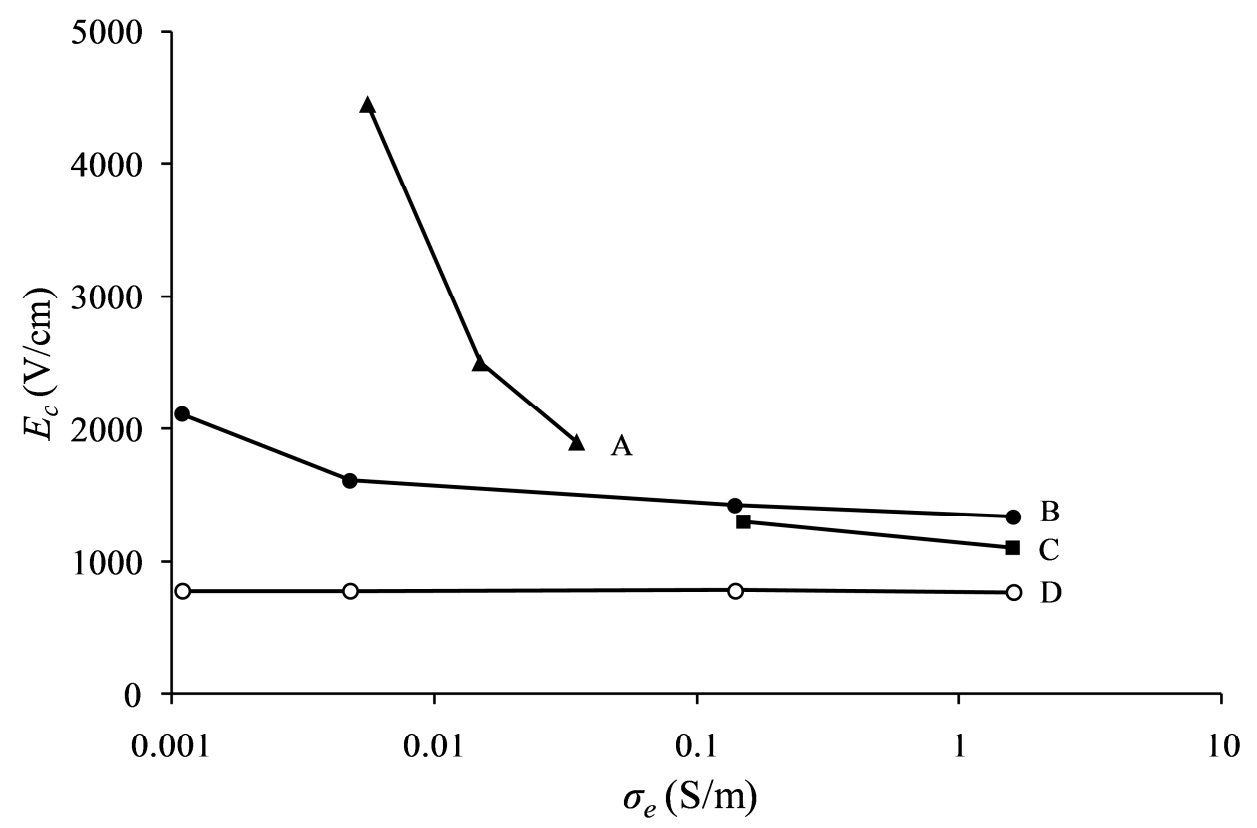

Fig.1 Results from experimental studies showing that the electric field magnitude required to achieve a specific electroporation effect, $E_{c}$, depends on the extracellular conductivity, $\sigma_{e}$. A: $50 \%$ green algae cells permeabilization after a single square pulse of $200 \mu \mathrm{s}$ as assessed by Serva Blue dye uptake ${ }^{1}$, B: $50 \%$ fibroblasts survival after a train of eight $100 \mu \mathrm{s}$ pulses ${ }^{10}$, C: definite level of gene expression (beta-galactosidase activity of $0.002 \mathrm{mU} / \mathrm{ng}$ DNA) in mesenchymal cells after a train of eight $100 \mu \mathrm{s}$ square pulses in a medium containing a plasmid encoding the lac $Z$ reporter gene ${ }^{11}$, D: $50 \%$ fibroblasts permeabilization after a train of eight $100 \mu$ s square pulses as assessed by bleomycin uptake ${ }^{10}$.

There are numerous evidences from experiments on cell suspensions ${ }^{16-18}$, on isolated cells ${ }^{19-21}$ and on artificial membranes ${ }^{22-24}$ that electroporation occurs when the transmembrane potential $\left(V_{m}\right)$ induced by the electric field reaches a specific threshold. The value of such threshold depends on the characteristics of the applied pulses (number, duration and shape) and also on how electroporation is assessed (e.g. by noticing an increase of membrane conductance, by detecting intracellular contents release or by observing cell lysis). Nevertheless most authors report $V_{m}$ threshold values in the range from $200 \mathrm{mV}$ to $1 \mathrm{~V}$.

Therefore, a plausible hypothesis that was proposed in the past $\mathrm{t}^{25,26}$ for explaining the dependence of $E_{c}$ on $\sigma_{e}$ can be phrased as follows: when an external electric field is applied, the induced transmembrane potential $\left(\Delta V_{m}\right)$ depends on the conductivity of the extracellular medium so that the threshold for the manifestation of the electroporation phenomenon can only be reached under some specific conditions. As it is reported in the appendix, in the case of a spherical cell it is possible to obtain analytical expressions for $\Delta V_{m}$ when an external electric is applied. And, taking into account realistic values for the geometry and for the dielectric parameters of the cells, such analytical expressions indeed show that the extracellular conductivity has an effect on the maximum value that $\Delta V_{m}$ can reach. However, the magnitude of such $\sigma_{e}$ effect on the calculated $\Delta V_{m}$ is insufficient for justifying the large dependences of $E_{c}$ on $\sigma_{e}$ that are observed experimentally (e.g. trace A in Fig. 1). Moreover, such analytical expressions are not able to predict the fact that $E_{c}$ sensitivity to $\sigma_{e}$ depends on the permeabilization level to be achieved, as illustrated in the cases B and D of Fig. 1. In other words, if it is assumed that $\sigma_{e}$ only has an 
impact on the electroporation phenomenon by modifying the minimum electric field at which electroporation can be manifested, then traces B and D should be proportional. Hence we believe that the model described by such analytical expressions needs to be improved with other phenomena in order to predict the experimentally observed behaviors. In particular, we propose to include the electroporation phenomenon (i.e. the formation of pores) in the model.

Here we hypothesize that the fact that membrane conductivity increases when electroporation occurs explains the observed phenomena. Reasoning behind this hypothesis is: 1) when an external voltage is applied, part of it drops at the membrane but the rest drops in the extracellular and intracellular media; 2) thus, voltage drop at the membrane, $V_{m}$, not only depends on its conductivity, $\sigma_{m}$, but also on the conductivity of extracellular and intracellular media $\left(\sigma_{e}\right.$ and $\sigma_{i}$ respectively); 3) when no electroporation occurs, membrane conductivity is very low and voltage drop at the membrane is almost independent on $\sigma_{e}$ and on $\sigma_{i}$, as described by the equations in the appendix; 4) however, when electroporation occurs because $V_{m}$ reaches the required threshold, the conductivity of the membrane increases abruptly ${ }^{27}$ and with a strong dependence on $\left.V_{m}{ }^{24} ; 5\right)$ as a consequence, voltage drop at the membrane is now significantly dependent on $\sigma_{e}$ and on $\sigma_{i}$ and in turn the membrane conductivity increase is also dependent on both conductivities. Then, since membrane conductivity reflects the permeabilization state at the time of the pulse, if is reasonable to expect that post-pulse effects of electroporation (e.g. uptake of molecular dyes, cell lysis, gene electrotransfer) will also be dependent on $\sigma_{e}$ and on $\sigma_{i}$.

The purpose of the numerical study presented here is to demonstrate that the above hypothesis can indeed justify the dependence of electroporation efficiency on $\sigma_{e}$ that is experimentally observed in the case of cells in suspension.

In the present report, the methods and the results sections are organized in two subsections. In both sections, the first subsection deals with the description and validation of a membrane conductivity model. The second subsection describes how that membrane conductivity model is employed in order to demonstrate that $\sigma_{e}$ can indeed have a significant effect on the amount of permeabilization.

\section{Materials and methods}

\subsection{Membrane conductivity model}

As a preliminary step, before addressing how extracellular conductivity influences permeabilization, we have selected a membrane conductivity model and we have assessed its performance by comparing simulated results with data from an experimental study in which the conductance of a dense cell suspension is measured during electroporation pulses ${ }^{28}$. Details about the model and its justification can be found in the results and discussion section. Here we simply specify some aspects concerning the numerical method we employed for validating the performance of the model.

We have performed numerical modeling of the conductance of a cell in suspension in a similar way to what is described in ${ }^{29}$. In particular, we have used a commercial finite element (FEM) software platform, COMSOL Multiphysics 3.5 (Stockholm, Sweden), to solve the steady- 
state problem depicted in Fig. 2. The parameter values and the dimensions of the FEM model are summarized in Table 1. We selected the values for $w$ and $h$ (dimensions of the measurement chamber) so that the volume of the cell is equivalent to $19 \%$ of the total volume of the chamber, as in the experimental study ${ }^{28}$. The membrane thickness in the model is unrealistically large (50 $\mathrm{nm}$ instead of $5 \mathrm{~nm}$ ) in order to facilitate FEM analysis. We verified that in the much slower simulations with a membrane thickness of $5 \mathrm{~nm}$ the results do not differ significantly from the results obtained with a $50 \mathrm{~nm}$ membrane.

Since the membrane conductivity depends on the electric field and, in turn, the electric field depends on the membrane conductivity, an iterative process is required to perform the simulation. In this iterative process, it is computed first the electric field distribution assuming $\sigma_{m}$ $=\sigma_{m 0}$, and then the equivalent $V_{m}$ at each point of the membrane is obtained as $V_{m}=\left|E_{\text {membrane }}\right| .50$ $\mathrm{nm}$, a new value for $\sigma_{m}$ is obtained according to Eq. 1 and the process is repeated until a stable solution is found. If in this iterative process it is modeled that the voltage at the electrodes is present since the first step, in some cases, the solution does not converge easily and oscillations in conductivity appear. Therefore, an alternative approach was tried here in order to facilitate convergence of the solution: the COMSOL simulation was in fact configured as a time transient simulation in which the applied voltage increased smoothly from $0 \mathrm{~V}$ to the final voltage and then remained at that final value until the end of the simulation. More specifically, the voltage raise consisted of a ramp function from time 0 to time 0.1 and the simulation finished at time 2 . It must be noted that here the time points are indicated without units (e.g. seconds) as they are in fact arbitrary; time has no physical meaning in the simulation, it is employed for facilitating the solving process. For each simulation it was verified that the solution was indeed stable and that no oscillations remained during the phase in which the applied voltage reached its final value.

Table 1 Model parameters for simulation of conductance of a single cell in suspension

\begin{tabular}{|c|c|c|}
\hline Symbol & Value & Definition, justification or source \\
\hline$\sigma_{m}$ & $\sigma_{m}=\sigma_{m 0}+K\left(e^{\beta\left|V_{m}\right|}-1\right)$ & Membrane conductivity model \\
\hline$\sigma_{m 0}$ & $2.5 \times 10^{-7} \mathrm{~S} / \mathrm{m}$ & $\begin{array}{c}\text { Membrane conductivity when } V_{m}=0, \text { form } \\
\text { thickness }=5 \mathrm{~nm}\end{array}$ \\
\hline$\sigma_{e l}$ & $1.58 \mathrm{~S} / \mathrm{m}$ & Extracellular conductivity, isotonic $\mathrm{NaCl}$ in ${ }^{28}$ \\
\hline$\sigma_{e 2}$ & $0.3 \mathrm{~S} / \mathrm{m}$ & $\begin{array}{c}\text { Extracellular conductivity, isotonic } 10 \% \mathrm{NaCl} / 90 \% \text { sucrose }{ }^{28} \text {, see } \\
\text { text for further justification }\end{array}$ \\
\hline$\sigma_{i}$ & $0.5 \mathrm{~S} / \mathrm{m}$ & Intracellular conductivity ${ }^{31}$ \\
\hline$\delta$ & $50 \mathrm{~nm}$ & Simulation membrane thickness, see text for justification \\
\hline$R$ & $2.8 \mu \mathrm{m}$ & Cell radius ${ }^{28}$ \\
\hline$V_{p}$ & 0 to $6 \mathrm{~V}$ & Applied potential, to produce fields from 0 to $\sim 6 \mathrm{kV} / \mathrm{cm}$ as in ${ }^{28}$ \\
\hline$w$ & $4.2 \mu \mathrm{m}$ & Simulation region width \\
\hline$h$ & $8.8 \mu \mathrm{m}$ & Simulation region height \\
\hline$n$ & 13320 & Number of elements in the mesh (FEM parameter) \\
\hline
\end{tabular}




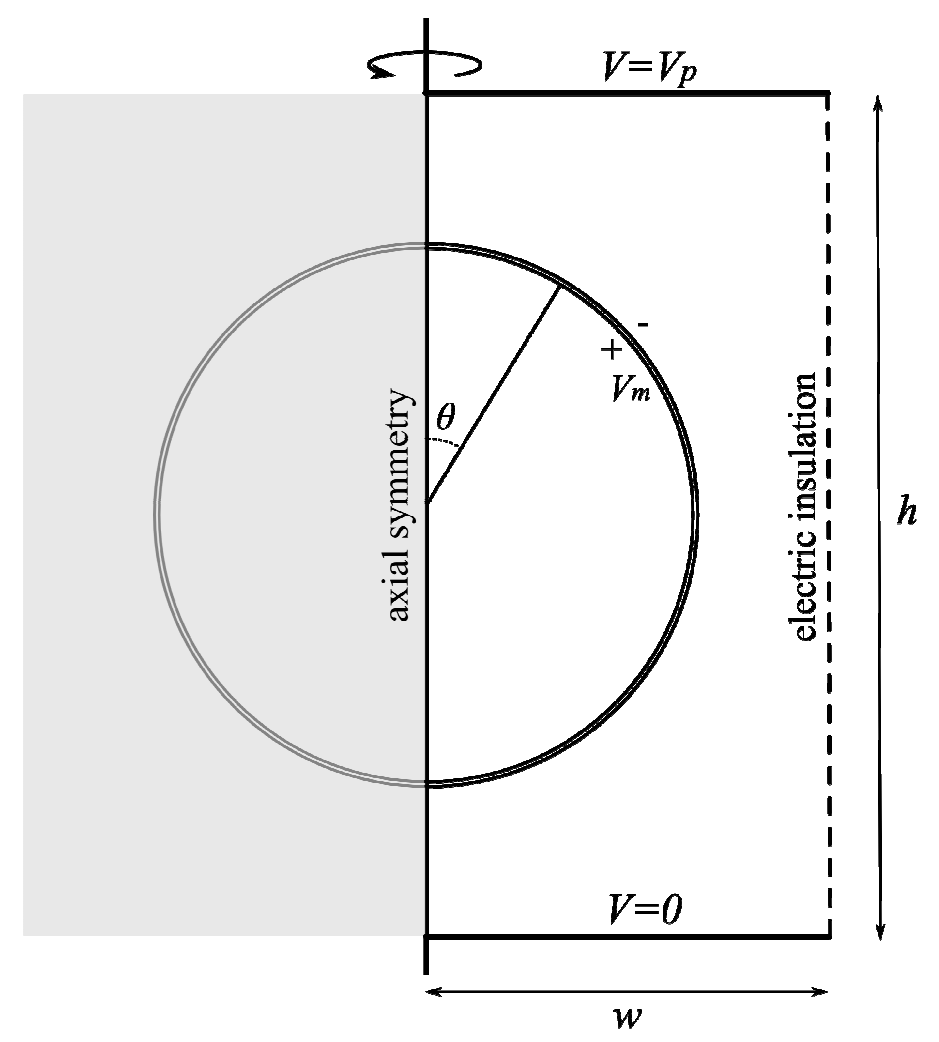

Fig. 2 Geometrical features of a model for a single cell in suspension. This model was solved by means of FEM and an axial symmetry was introduced in order to reduce computation time. The conductivities of the extracellular and intracellular media are constant whereas the conductivity of the membrane $\left(\sigma_{m}\right)$ depends on the local electric field which in turn depends on $\sigma_{m}$. The volume fraction of the cell is approximately $20 \%$, as in ${ }^{28}$.

\subsection{Modeling membrane conductivity increase dependence on extracellular conductivity}

For studying the influence of extracellular conductivity on membrane conductivity increase during electroporation, we have employed the previous numerical model with a minor modification of the geometrical dimensions (see Table 2); only the width, $w$, and the height, $h$, of the chamber are slightly larger in order to ensure electric field uniformity at distant points from the cell. Further details about the model and its justification, particularly the reason why we change the value of the constant $K$ in the membrane conductivity model for each extracellular conductivity value, are given in the results and discussion section. 
Table 2 Model parameters for simulation of membrane conductivity dependence on extracellular conductivity in the case of a single cell in suspension

\begin{tabular}{ccc}
\hline Symbol & Value & Definition, justification or source \\
\hline$\sigma_{m}$ & $\sigma_{m}=\sigma_{m 0}+K\left(e^{\beta\left|V_{m}\right|}-1\right)$ & Membrane conductivity \\
$\beta$ & 16 & Constant for $\sigma_{m}$ \\
$K$ & $10^{-9} \times: 3.75$ for $\sigma_{e l}, 1.5$ for & Constant for $\sigma_{m}$ \\
& $\sigma_{e 2}, 1.275$ for $\sigma_{e 3}$ and & \\
$\sigma_{m 0}$ & 1.2525 for $\sigma_{e 4}$ & Membrane conductivity when $V_{m}=0$, see Table 1 \\
$\sigma_{e 1}$ & $2.5 \times 10^{-7} \mathrm{~S} / \mathrm{m}$ & Extracellular conductivity \\
$\sigma_{e 2}$ & $1 \mathrm{~S} / \mathrm{m}$ & Extracellular conductivity \\
$\sigma_{e 3}$ & $0.1 \mathrm{~S} / \mathrm{m}$ & Extracellular conductivity \\
$\sigma_{e 4}$ & $0.01 \mathrm{~S} / \mathrm{m}$ & Extracellular conductivity \\
$\sigma_{i}$ & $0.001 \mathrm{~S} / \mathrm{m}$ & Intracellular conductivity 31 \\
$\delta$ & $0.5 \mathrm{~S} / \mathrm{m}$ & Cell radius 28 \\
$R$ & $50 \mathrm{~nm}$ & Simulation membrane thickness, see text for justification \\
$V_{p}$ & $2.8 \mu \mathrm{m}$ & Applied potential, to produce fields from 0 to $5 \mathrm{kV} / \mathrm{cm}$ \\
$w$ & $0 \mathrm{to} 5 \mathrm{~V}$ & Simulation region width \\
$h$ & $5 \mu \mathrm{m}$ & Simulation region height \\
$n$ & $10 \mu \mathrm{m}$ & Number of elements in the mesh (FEM parameter)
\end{tabular}

\section{Results and discussion}

\subsection{Membrane conductivity model}

Membrane conductivity models for electroporation range from simple voltage-sensitive switches 32 to complicated models that are supposed to be able to quantify the total number of pores and their dimensions ${ }^{31,33}$. Here we have decided to minimize assumptions regarding the electroporation process and the nature of the pores and we have chosen to employ a simple model that can be described with a single continuous function:

$\sigma_{m}=\sigma_{m 0}+K\left(e^{\beta\left|V_{m}\right|}-1\right)$

where $\sigma_{m 0}$ is the conductivity of the membrane for $V_{m}=0$ (before electroporation occurs) and $K$ and $\beta$ are two constants that describe how membrane conductivity increases as the transmembrane potential increases.

Under the hypothesis that membrane conductivity rise is due to pores, we can rewrite Eq. 1 as:

$\sigma_{m}=\sigma_{m 0}+\sigma_{\text {pores }}\left(\lambda\left(e^{\beta\left|V_{m}\right|}-1\right)\right)$

where $\sigma_{\text {pores }}$ would be the conductivity of the solution filling the pores, $\lambda$ would be another constant and the term 
$S_{p}=\lambda\left(e^{\beta\left|V_{m}\right|}-1\right)$

would represent the relative area of the pores. Therefore, the constant $K$ in Eq. 1 would depend on $\sigma_{\text {pores }}$.

For determining the value of the constants $K$ and $\beta$ we have made use of data reported in an experimental study in which the conductivity of a dense cell suspension was measured during electroporation for multiple pulse amplitudes ${ }^{28}$. In particular, we manually adjusted the value of the constants $K$ and $\beta$ so that our model for a cell in suspension (see paragraphs below and Table 1 and Fig. 2) provided conductance measurements that matched the experimental measurements at $2 \mu \mathrm{s}$ after the beginning of the pulse. This initial adjustment was done for data obtained in isotonic saline $\left(\mathrm{NaCl} 0.9 \%, \sigma_{e}=1.58 \mathrm{~S} / \mathrm{m}^{28}\right)$. Then, we tried to use the same membrane conductivity model for fitting experimental results obtained in isotonic $10 \% \mathrm{NaCl} / 90 \%$ sucrose $\left(\sigma_{e}=0.3 \mathrm{~S} / \mathrm{m}\right)$ and we discovered that experimental data seemed better approximated if $K$ was halved (Fig. 3); which would be in agreement with Eq. 2 under the reasonable assumption that $\sigma_{\text {pores }}$ depends on $\sigma_{e}$, as justified below.

Ionic species such as $\mathrm{Na}^{+}, \mathrm{Cl}^{-}$and $\mathrm{K}^{+}$have diffusion coefficients in water of about $1.5 \times 10^{-}$ ${ }^{9} \mathrm{~m}^{2} / \mathrm{s}$. ${ }^{34}$ This implies that in a pore with a length of $5 \mathrm{~nm}$ (cell membrane thickness) the ionic content will be a good mixture of the ionic contents of both compartments in less than $2 \mu \mathrm{s}$ after pore creation (further discussed and justified some paragraphs below). Therefore, the conductivity of the pores can be assumed to be:

$$
\sigma_{\text {pores }}=\frac{\sigma_{e}+\sigma_{i}}{2}
$$

And the value of $K$ for $\sigma_{e 2}, K_{2}$, relative to the value of $K$ for $\sigma_{e l}, K_{l}$, is:

$$
K_{2}=\frac{\sigma_{\text {pores } 2}}{\sigma_{\text {pores } 1}} K_{1}=\frac{\sigma_{e 2}+\sigma_{i}}{\sigma_{e 1}+\sigma_{i}} K_{1}
$$

In the above case $\left(\sigma_{i}=0.5 \mathrm{~S} / \mathrm{m}, \sigma_{e l}=1.58 \mathrm{~S} / \mathrm{m}, \sigma_{e 2}=0.3 \mathrm{~S} / \mathrm{m}\right)$ the value of $K_{2}$ would be $\sim 0.4 K_{l}$ which is reasonably close to the value obtained by fitting the experimental data $\left(K_{2}=0.5 K_{l}\right)$. As a consequence, for modeling the membrane conductivity increase dependence on extracellular conductivity we have decided to follow the model described in Eq. 1 with a $K$ value that depends on the extracellular conductivity as described in Eq. 5. Actually, we have rounded $\sigma_{e l}$ to $1.5 \mathrm{~S} / \mathrm{m}$ so that the expression we used for $K$ is simplified:

$$
K=5 \times 10^{-9} \frac{\sigma_{e}+\sigma_{i}}{2}
$$

As it can be observed in Fig. 3, with the exponential model for $\sigma_{m}\left(V_{m}\right)$ described by Eq. 1 it is possible to reproduce very accurately the conductance measurements experimentally obtained from a cell suspension subjected to electroporation. ${ }^{28}$ 
A remarkable phenomenon that should be pointed out first is that whereas membrane conductivity rise as a function of $V_{m}$ is extremely abrupt (model plotted in a logarithmic scale in the insert of Fig. 3), suspension conductance increase as a function of the applied field magnitude is much smoother. This behavior, which is also observed in living tissues ${ }^{35}$, is linked to the main hypothesis of the present study: as $|E|$ increases $V_{m}$ also tends to rise which causes an increase of $\sigma_{m}$ which in turn tends to lessen the increase of $V_{m}$ in a sort of negative feedback fashion.

As it has been mentioned, we manually adjusted the constants $K$ and $\beta$ of Eq. 1 for matching the simulated data (bottom thick continuous line in Fig. 3) to the experimental data measured in saline $\left(\sigma_{e}=1.58 \mathrm{~S} / \mathrm{m}\right)$. This adjustment yielded: $K=5 \times 10^{-9}$ and $\beta=16$. Then, by using the same model and constants for $\sigma_{m}$, we tried to fit the experimental data obtained in isotonic $10 \% \mathrm{NaCl} / 90 \%$ sucrose with the extracellular conductivity value reported by the authors of the experimental study ${ }^{28}, \sigma_{e}=0.158 \mathrm{~S} / \mathrm{m}$. However, such fitting was only partially successful, even when we tried by modifying the values of the constants $K$ and $\beta$, we were not able to reproduce exactly the shape of the cell suspension conductance as a function of $|E|$ (data not reported here). On the other hand, by modifying the value $\sigma_{e}$ used in the simulations we noticed that we were able to get curves that matched the pattern of the experimental data. In particular, a value for $\sigma_{e}$ of $0.3 \mathrm{~S} / \mathrm{m}$ yielded an almost perfect matching. Therefore, we are inclined to assume that the actual conductivity of the extracellular medium in ${ }^{28}$ was $0.3 \mathrm{~S} / \mathrm{m}$ (instead of $\left.0.158 \mathrm{~S} / \mathrm{m}\right)$. In favor of this assumption we can point out at least another fact besides our simulations results: according to ${ }^{28}$, media conductivities were measured before the cell suspensions were prepared, not afterwards, and then, since an outflow of intracellular ions would be favorable during suspension storage because of the concentration gradient (time not indicated in ${ }^{28}$ ), it is reasonable to expect lower values for $\sigma_{i}$ and higher values for $\sigma_{e}$ than the initially measured ones $\left(\sigma_{i}=0.5 \mathrm{~S} / \mathrm{m}, \sigma_{e}=0.158\right.$ $\mathrm{S} / \mathrm{m}$ ). As a matter of fact, we verified that by decreasing the value of $\sigma_{i}$ we also obtained almost perfect fittings without requiring values of $\sigma_{e}$ as high as $0.3 \mathrm{~S} / \mathrm{m}$ (data not reported here). Moreover, dense concentrations of microorganisms in suspension, as it is the case, are known to increase significantly the conductivity of the medium due to metabolic byproducts ${ }^{36}$. Hence the assumption that $\sigma_{e}$ is larger than $0.158 \mathrm{~S} / \mathrm{m}$ is quite plausible, together with a possible decrease of $\sigma_{i}$. However, for the sake of simplicity, in our simulations we have decided to keep $\sigma_{i}$ constant and to assume that $\sigma_{e}$ for isotonic $10 \% \mathrm{NaCl} / 90 \%$ sucrose is $0.3 \mathrm{~S} / \mathrm{m}$. With these values, the fitting was further improved when $K$ was $2.5 \times 10^{-9}$ instead of $5 \times 10^{-9}$ (see Fig. 3). As pointed out above, this improvement by halving $K$ is in agreement with the model described by Eq. 4 in which the conductivity of the pores, $\sigma_{p o r e s}$, is the average value of $\sigma_{e}$ and $\sigma_{i}$. 


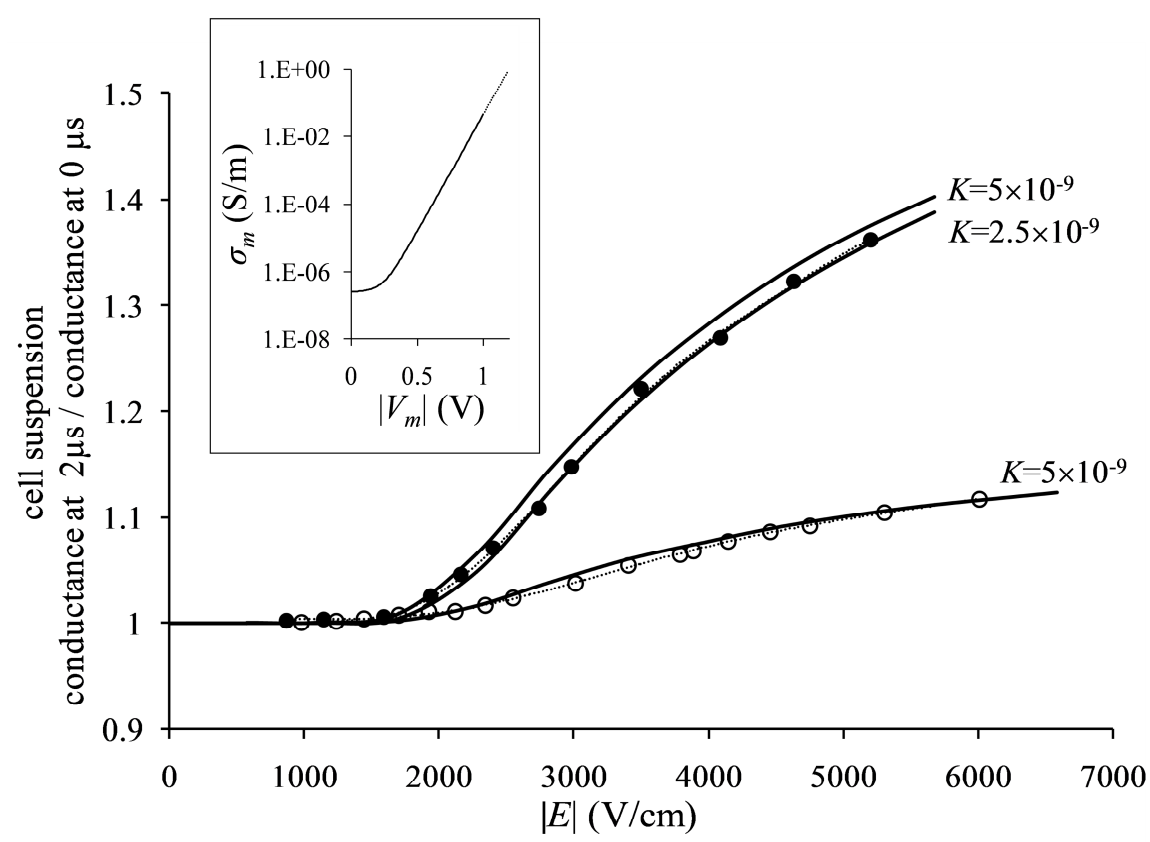

Fig. 3 Experimental and simulated cell suspension relative conductance (conductance at $2 \mu$ s / conductance before pulse). Experimental data is from ${ }^{28}$ when the extracellular medium is isotonic saline (0) and when the extracellular medium is isotonic $10 \% \mathrm{NaCl} / 90 \%$ sucrose $(\bullet)$. The simulated results (continuous lines) are based on the model presented in Fig. 2 when the membrane conductivity behaves as it is described in Eq. 3 (see insert). The parameters $\sigma_{m 0}$ and $\beta$ (adjusted for isotonic saline experimental data) are equal for all simulated results whereas $K$ has two different values (see text). The two thin dashed lines are sixth order polynomial approximations of the experimental data.

Fig. 4 shows that simulated transmembrane potential along the cell behaves according to Eq. 9 in the appendix (i.e. $V_{m}$ proportional to $\cos (\theta)$ ) when the electric field magnitude is 1500 $\mathrm{V} / \mathrm{cm}$. On the other hand, for larger field magnitudes $(3000 \mathrm{~V} / \mathrm{cm}$ and $6000 \mathrm{~V} / \mathrm{cm}), V_{m}$ saturates at values around $1 \mathrm{~V}$ at the cell poles facing the hypothetical location of the electrodes. This phenomenon is the consequence of a large increase in membrane conductivity at the areas where $V_{m}$ is above a specific threshold (around $1 \mathrm{~V}$ in this case) and it has been experimentally observed in actual cells by employing voltage-sensitive dyes ${ }^{21}$. 


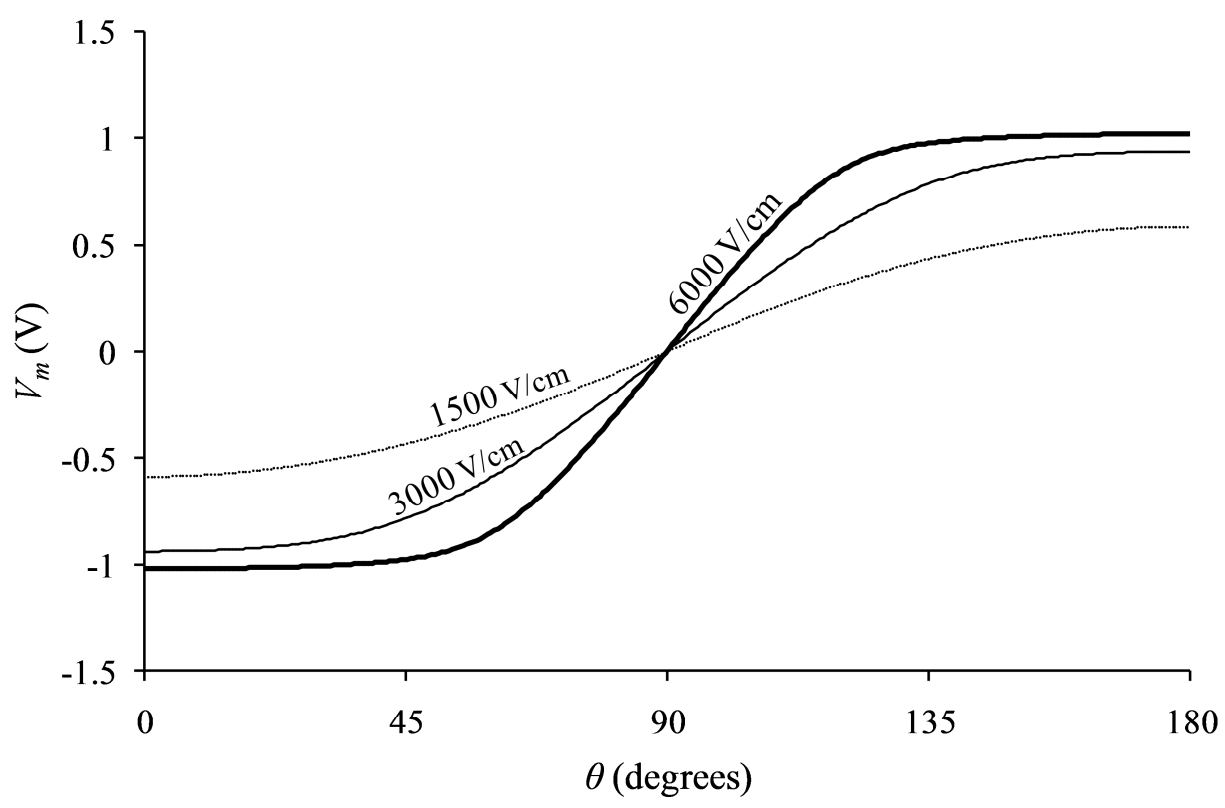

Fig. 4 Simulated transmembrane potential $\left(V_{m}\right)$ from the model displayed in Fig. 2 for three different electric field magnitudes. For $1500 \mathrm{~V} / \mathrm{cm}\left(V_{p}=1.32 \mathrm{~V}\right)$ the transmembrane potential is proportional to $\cos (\theta)$ as predicted by Eq. 1. For larger fields, $3000 \mathrm{~V} / \mathrm{cm}(2.64 \mathrm{~V})$ and $6000 \mathrm{~V} / \mathrm{cm}(5.28 \mathrm{~V}), V_{m}$ saturates at the poles facing the electrodes due to the increase of membrane conductivity caused by electroporation, as experimentally observed in ${ }^{21}$.

In our model we have specified that the conductivity of the pores $\left(\sigma_{\text {pores }}\right)$ is the average value of $\sigma_{e}$ and $\sigma_{i}$. This is done under the assumption that anions and cations diffuse sufficiently rapidly across the pores so that a perfect ionic mixture is achieved within the pores in less than 2 $\mu \mathrm{s}$. This assumption is reasonably valid if the diffusion coefficient for ions inside the pores is similar to that of ions in aqueous solutions $\left(D \sim 1.5 \times 10^{-9} \mathrm{~m}^{2} / \mathrm{s}\right)$. However, diffusion across pores may be hindered and it is quite plausible that the diffusion coefficients of ions in the pores is 10 or 100 times lower than in bulk water ${ }^{37}$. Therefore, we decided to check whether the assumption of perfect mixture at $2 \mu \mathrm{s}$ was realistic. For that purpose, we built a simple COMSOL model (Fig. 5) for computing the equivalent conductivity of the pores medium $2 \mu$ s after pore creation. Equivalent conductivity of the pore medium was computed as $\sigma_{\text {pores }}=G_{\text {pore }} \times$ (pore length/pore section), where $G_{\text {pore }}$ is the conductance assessed between both openings of the pore. As expected, at $2 \mu \mathrm{s}$, for the lowest diffusion coefficient $\left(1.5 \times 10^{-11} \mathrm{~m}^{2} / \mathrm{s}\right)$ concentration distribution within the pore is far from being a perfect mixture (data not reported). However, as it can be noticed in Table 3 , equivalent pore medium conductivity does not diverge drastically from the perfect mixture case $\left(D=\infty \mathrm{m}^{2} / \mathrm{s}\right)$. Hence we conclude that the assumption of perfect mixture is realistic for computing $\sigma_{\text {pores }}$ in the context of the present study (we did check that considering $\sigma_{\text {pores }}=0.11 \mathrm{~S} / \mathrm{m}$, for $\sigma_{e}=0.01 \mathrm{~S} / \mathrm{m}$, instead of $\sigma_{\text {pores }}=0.2505 \mathrm{~S} / \mathrm{m}$ did not have a significant qualitative impact on the results described in next section). 


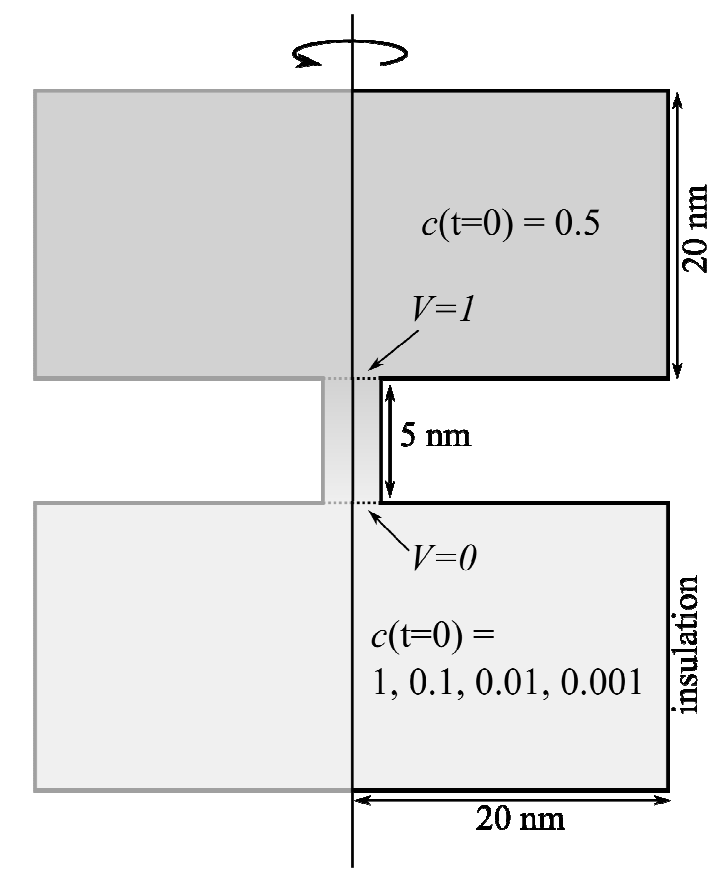

Fig. 5 Geometry of the model employed for verifying that mere diffusion would be sufficient for assuring that conductivity of the medium in a pore would be close to the average value of $\sigma_{e}$ and $\sigma_{i}$ just $2 \mu$ s after pore creation. The initial concentration, $c$, in the intracellular medium is $0.5 \mathrm{~mol} / \mathrm{m}^{3}$ whereas various initial concentrations are tried for the extracellular medium. Pore conductance, $G_{p o r e}$, is assessed between both openings of the pore assuming that a concentration of $1 \mathrm{~mol} / \mathrm{m}^{3}$ has a conductivity of $1 \mathrm{~S} / \mathrm{m}$. Equivalent conductivity of pore medium, $\sigma_{\text {pores }}$, is calculated as $\sigma_{\text {pores }}=G_{\text {pore }} \times$ pore length/pore section. Results at $2 \mu$ s are shown in Table 3.

Table 3 Equivalent conductivity of the medium inside the pores at $2 \mu$ s after pore creation for different diffusion coefficients and different extracellular conductivities

\begin{tabular}{cccccc}
\hline & $\sigma_{i}(\mathrm{~S} / \mathrm{m})$ & 0.5 & 0.5 & 0.5 & 0.5 \\
$\sigma_{e}(\mathrm{~S} / \mathrm{m})$ & 1 & 0.1 & 0.01 & 0.001 \\
\hline \multirow{3}{*}{$\sigma_{\text {pores }}(\mathrm{S} / \mathrm{m})$} & $D=\infty \mathrm{m}^{2} / \mathrm{s}$ & 0.75 & 0.3 & 0.255 & 0.2505 \\
& $D=1.5 \times 10^{-9} \mathrm{~m}^{2} / \mathrm{s}$ & 0.74 & 0.29 & 0.24 & 0.23 \\
& $D=1.5 \times 10^{-11} \mathrm{~m}^{2} / \mathrm{s}$ & 0.72 & 0.25 & 0.13 & 0.11 \\
\hline
\end{tabular}

Instead of assuming that pores are filled with a mixture of extracellular and intracellular ionic solutions due to diffusion, an alternative physical model for the increase of membrane conductivity could be the following: the electric field creates pores or water channels in the membrane, as predicted by molecular dynamics simulations ${ }^{38,39}$, and then extracellular and intracellular ions are pulled and pushed through those pores because of the same electric field. Therefore, the equivalent membrane conductivity would be proportional to the area of the pores (which again we can imagine to be described by Eq. 3) and to the amount of free ions in the media in contact with the membrane. Ionic contents of the pores would not be relevant as extracellular and intracellular ions would be provided at sufficient speed: for an electric field magnitude in the order of $1000 \mathrm{~V} / \mathrm{cm}$, monovalent ions travel at a velocity of about $1 \mathrm{~cm} / \mathrm{s}$ which implies that ions can be pushed, or pulled, through the pores in less than $0.5 \mu \mathrm{s}$ (particularly if we take into account that fields in the vicinity of the pores will actually be much larger than $1000 \mathrm{~V} / \mathrm{cm}$ ). Hence, since the amount of free ions in a medium is roughly 
proportional to its conductivity, it turns out that both physical descriptions would in fact produce the same result in terms of electrical modeling: the increase of conductivity for a given permeabilization level (given by Eq. 3) is roughly proportional to the average value of value of $\sigma_{e}$ and $\sigma_{i}$.

Finally, we want to point out in this section that other functions besides exponential functions could be also employed for modeling membrane conductivity. The only requirement for matching accurately the cell suspension conductivity experimental data seems to be an abrupt, but finite, increase of conductivity when $V_{m}$ reaches values close to $1 \mathrm{~V}$. The pattern of the dependence of cell suspension conductivity on electric field magnitude seems to be mostly determined by $\sigma_{e}, \sigma_{i}$ and the geometry rather than by the shape of the function modeling $\sigma_{m}$. For instance, in Fig. 6 it can be noticed that the following power function also reproduces with high fidelity the experimental data:

$\sigma_{m}=\sigma_{m 0}+0.7\left(V_{m}^{12}\right)$

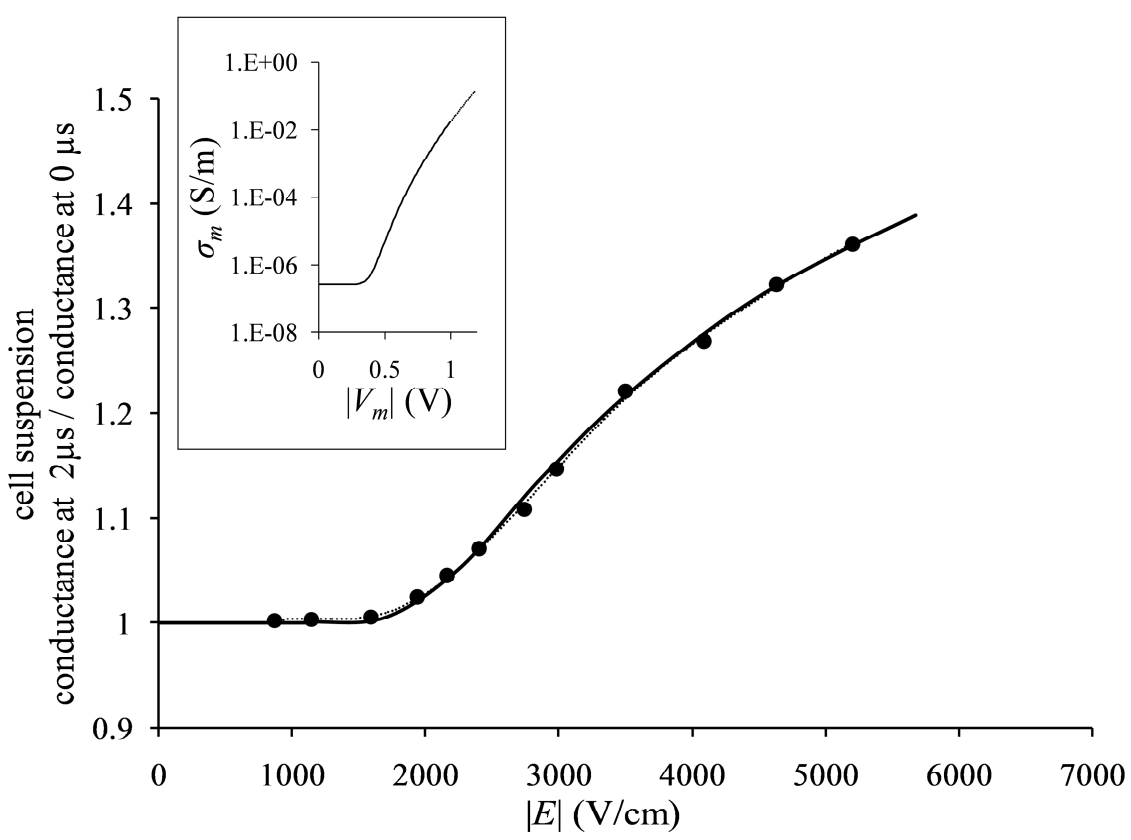

Fig. 6 Experimental and simulated cell suspension relative conductance (conductance at $2 \mu$ s / conductance before pulse) when the model employed for membrane conductivity consists of a power function (see insert) instead of an exponential function (see Fig. 3). Experimental data is from ${ }^{28}$ when the extracellular medium is isotonic $10 \%$ $\mathrm{NaCl} / 90 \%$ sucrose $(\bullet)$. Simulated results are depicted with a continuous line. The thin dashed line is a sixth order polynomial approximation of the experimental data. 


\subsection{Permeabilization dependence on medium conductivity}

As expected, the simulated average membrane conductivity, $\bar{\sigma}_{m}$, (Fig. 7) increases as field magnitude increases above a certain threshold ( $\sim 500 \mathrm{~V} / \mathrm{cm}$ in Fig. 7). For field magnitudes between $1000 \mathrm{~V} / \mathrm{cm}$ and $2500 \mathrm{~V} / \mathrm{cm}$ the rise is quite steep but afterwards it moderates due to the mitigation of $V_{m}$ increase mentioned before. Nevertheless, the most remarkable observable fact in Fig. 7 is that membrane conductivity increase depends significantly on the extracellular conductivity. For instance, for a given membrane conductivity value of $1 \times 10^{-4} \mathrm{~S} / \mathrm{m}$ it can be observed that the electric field magnitude required to reach it is slightly lower than $2000 \mathrm{~V} / \mathrm{cm}$ if $\sigma_{e}$ is $1 \mathrm{~S} / \mathrm{m}$, slightly larger than $2000 \mathrm{~V} / \mathrm{cm}$ if $\sigma_{e}$ is $0.1 \mathrm{~S} / \mathrm{m}$, of about $3000 \mathrm{~V} / \mathrm{cm}$ if $\sigma_{e}$ is $0.01 \mathrm{~S} / \mathrm{m}$ and much larger than $5000 \mathrm{~V} / \mathrm{cm}$ (out of scale) if $\sigma_{e}$ is $0.001 \mathrm{~S} / \mathrm{m}$. Hence this is a first indication that the effect of electroporation indeed depends on the extracellular conductivity. Nevertheless, it is more interesting to analyze what happens in terms of permeabilization.

Following the pore model described by Eqs. 2 and 3, the obtained average conductivity values can be scaled according to the following equation so that the average relative area of the pores is obtained:

$\bar{S}_{p}=\frac{\bar{\sigma}_{m}-\sigma_{m 0}}{\sigma_{\text {pores }}}$

where $\sigma_{m 0}$ is the membrane conductivity when transmembrane potential is $0 \mathrm{~V}$, and $\sigma_{\text {pores }}$ is the equivalent conductivity of the medium filling the pores as described by Eq. 6 . The result of such translation (Fig. 8) shows that the amount of permeabilization is indeed also dependent on the extracellular conductivity. In particular, if we plot the required electric field magnitude, $E_{c}$, required to achieve three different levels of permeabilization at $2 \mu$ s (relative pores area of $1 \times 10^{-}$ $5,3 \times 10^{-5}$ and $1 \times 10^{-4}$ ) it can be observed that the resulting curves (Fig. 9) resemble the ones plotted in Fig. 1 from experimental data. The critical field, $E_{c}$, is practically independent of $\sigma_{e}$ when $\sigma_{e}$ has values of about $0.1 \mathrm{~S} / \mathrm{m}$ and $1 \mathrm{~S} / \mathrm{m}$, as it is the case for traces B and D in Fig. 1. For lower extracellular conductivities $\left(\sigma_{e}<0.1 \mathrm{~S} / \mathrm{m}\right)$ modeled $E_{c}$ may be significantly dependent on $\sigma_{e}$, as it is also the case for traces A and B in Fig. 1. More interestingly, such significant dependence of $E_{c}$ on $\sigma_{e}$ is dependent on the chosen amount of permeabilization: for a relative pores area of $1 \times 10^{-5}$ (i.e. pores occupy $0.001 \%$ of membrane area) the value of $E_{c}$ is practically independent of $\sigma_{e}$ whereas for a relative pores area of $1 \times 10^{-4}$ the value of $E_{c}$ is strongly dependent on $\sigma_{e}$. This last observation would explain what was noticed in ${ }^{10}$ (i.e. traces B and D in Fig. 1): the lower amount of permeabilization required in $\mathrm{D}$ (cell death assessed after reversible electroporation followed by uptake of cytotoxic drug) is almost insensitive to $\sigma_{e}$ whereas the higher amount of permeabilization required in B (direct cell death caused by irreversible electroporation) is significantly dependent on $\sigma_{e}$.

Our initial hypothesis was that the experimentally observed dependence of electroporation effects on the conductivity of the extracellular medium could be explained numerically as being the result of the dependence of membrane conductivity, $\sigma_{m}$, on the transmembrane potential, $V_{m}$. In the present study, by applying an exponential model for $\sigma_{m}\left(V_{m}\right)$ we have provided evidences that support such hypothesis. Nevertheless we want to point out that other models for $\sigma_{m}\left(V_{m}\right)$ 
would also yield the same conclusion. For instance, in the appendix it is reported that a sigmoidal model for $\sigma_{m}\left(V_{m}\right)$ produces a similar qualitative behavior for the dependence of $E_{c}$ on $\sigma_{e}$.

The exponential model we applied for $\sigma_{m}\left(V_{m}\right)$ includes a term, $\sigma_{m 0}$, for modeling leakage currents through the non-selective ion channels of the membrane. Neither the selective ion channels nor the voltage-gated channels are taken into account in our model. This simplification has also been performed in previous modeling studies ${ }^{31,33}$. Those non-linear ion channels are very relevant and abundant in excitable cells (e.g. neurons and myocytes) but their presence is very limited in other cells, such as the RBCs considered in our study. Nevertheless, even in the case of excitable cells, the contribution of those non-linear ion channels is not very significant in terms of conductance and in the context of the present study: during an action potential, the membrane conductivity of an axon membrane ${ }^{41}$ can only increase up to about $1 \times 10^{-6}[\mathrm{~S} / \mathrm{m}]$, a value that would be close to the average membrane conductivity starts to be significant according to our model (see Fig. 7). Therefore, if those selective and voltage-gated ion channels had been modeled in the current study, their effect on our hypothesis would had been negligible since the dependence of pores area on extracellular conductivity is observed for larger electric fields (Fig. 8)

Finally, we would like to discuss briefly the experimental data shown in Fig. 1 regarding plasmid transfection (trace $\mathrm{C}$ ): the dependence of the level of expression on the extracellular conductivity is not predicted precisely by what we have concluded before regarding the amount of permeabilization; note that traces for $\sigma_{e}=1 \mathrm{~S} / \mathrm{m}$ and $\sigma_{e}=0.1 \mathrm{~S} / \mathrm{m}$ barely diverge in Fig. 8 whereas trace $\mathrm{C}$ in Fig.1 indicates that there is a significant difference in the level of expression for both conductivities. Various reasons could easily explain such discrepancy: 1) our modeling was intended for a single pulse, not for multiple sequential pulses, as it is normally the case in actual electroporation processes, and pulse repetition is known to have a memory effect on the conductivity of the membranes ${ }^{42}$; 2 ) furthermore, our modeling only considers what happens during the first instants $(2 \mu \mathrm{s})$ of the pulse, when it is believed that most small pores are created ${ }^{31}$, but does not predict how the pores could evolve afterwards as a function of the extracellular conductivity. Nevertheless, it must be pointed out that transport of DNA into the cell by electroporation is believed to be more complicated that the mere diffusion of the DNA constructs into the cells after membrane permeabilization. In particular, it has been proposed that electrophoretic forces play a significant role ${ }^{43-45}$. If that is the case, then our model could also explain the experimental observations: whereas the pores areas (Fig. 8) for both extracellular conductivities are very similar, the conductivities are significantly different (Fig. 9) and therefore one could expect that electrophoretic forces would be also significantly different. In particular, as observed in Fig 1, plasmid transfection for $\sigma_{e}=0.1 \mathrm{~S} / \mathrm{m}$ would require a significantly higher field than the same level of transfection for $\sigma_{e}=1 \mathrm{~S} / \mathrm{m}$. 


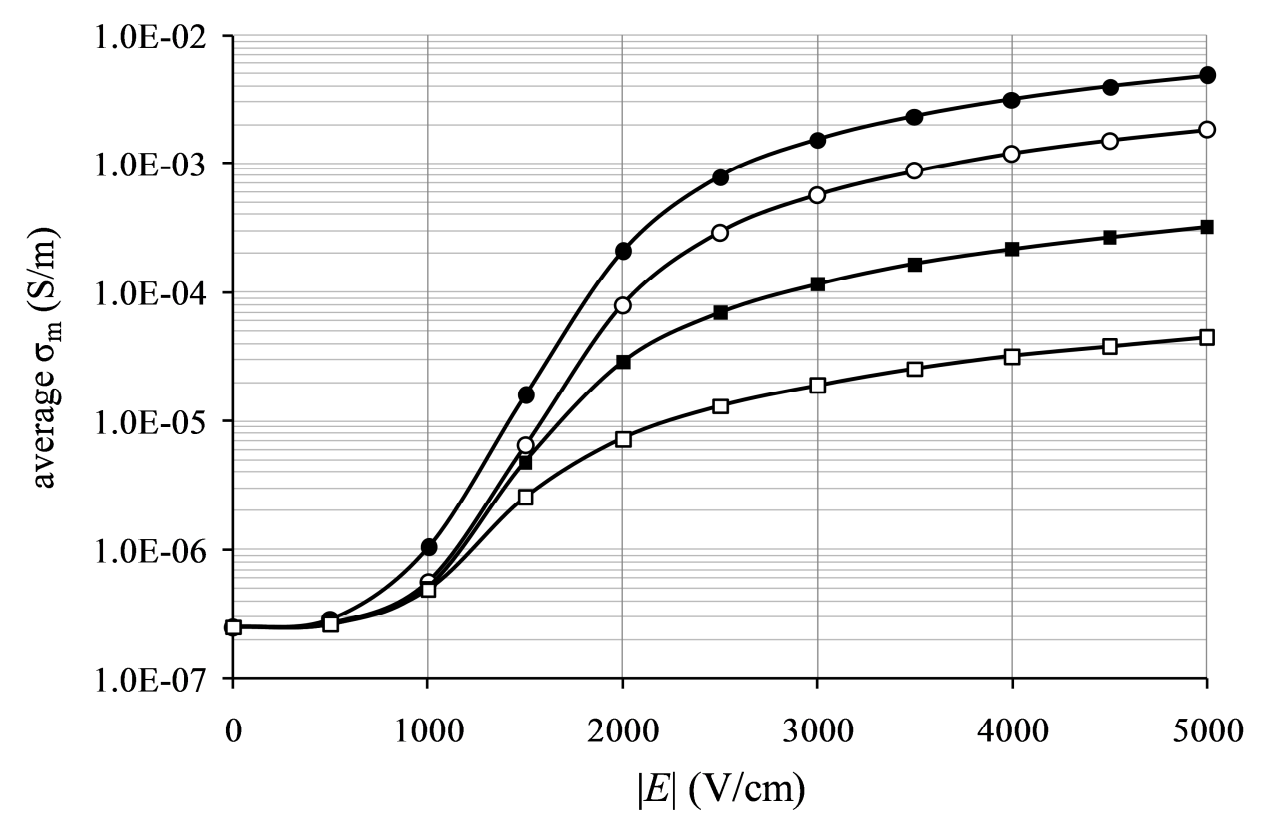

Fig. 7 Modeled average conductivity of cell membrane when $\sigma_{e}=1 \mathrm{~S} / \mathrm{m}(\bullet), \sigma_{e}=0.1 \mathrm{~S} / \mathrm{m}(\circ), \sigma_{e}=0.01 \mathrm{~S} / \mathrm{m}(\boldsymbol{\square})$ and $\sigma_{e}=0.001 \mathrm{~S} / \mathrm{m} \mathrm{( \square ).}$

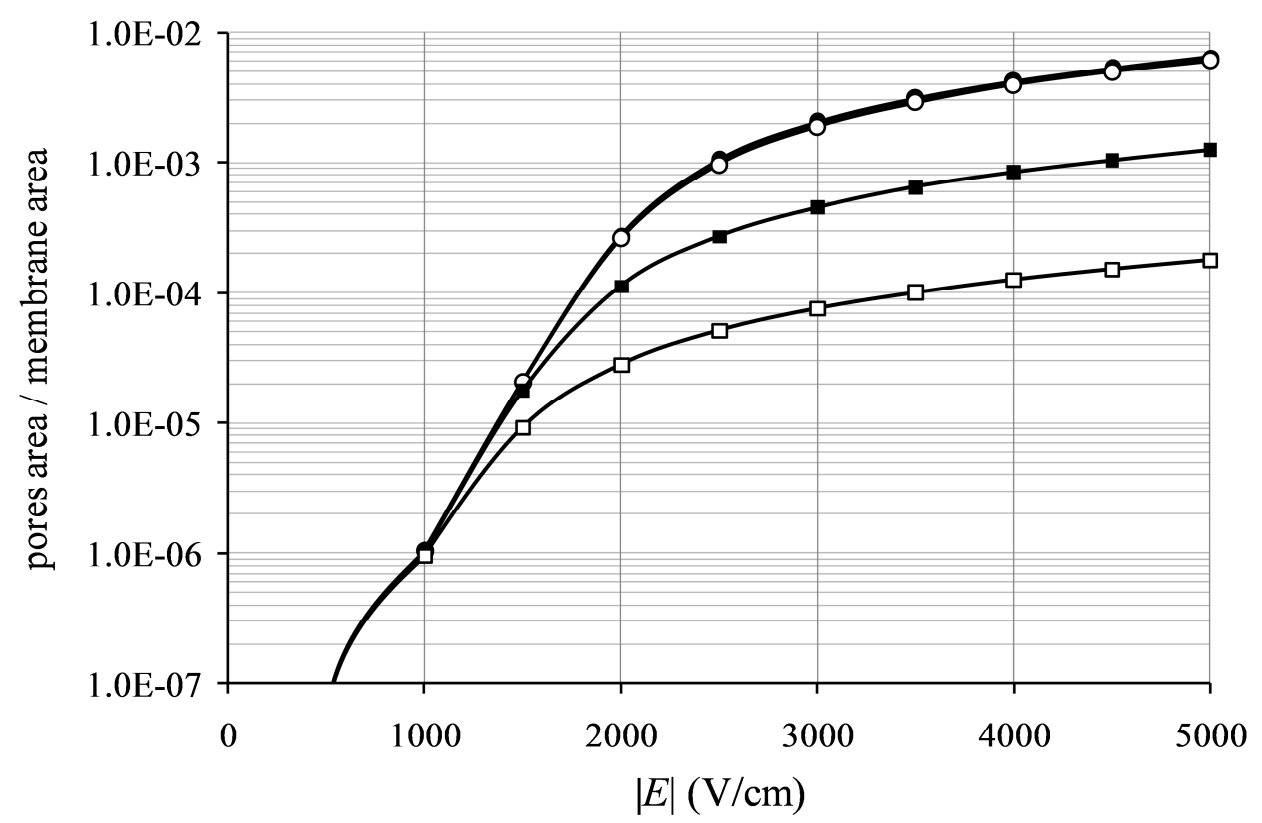

Fig. 8 Modeled relative area of the pores (total are of pores / membrane area) when $\sigma_{e}=1 \mathrm{~S} / \mathrm{m}(\bullet), \sigma_{e}=0.1 \mathrm{~S} / \mathrm{m}(\circ)$, $\sigma_{e}=0.01 \mathrm{~S} / \mathrm{m}(\square)$ and $\sigma_{e}=0.001 \mathrm{~S} / \mathrm{m}(\square)$. 


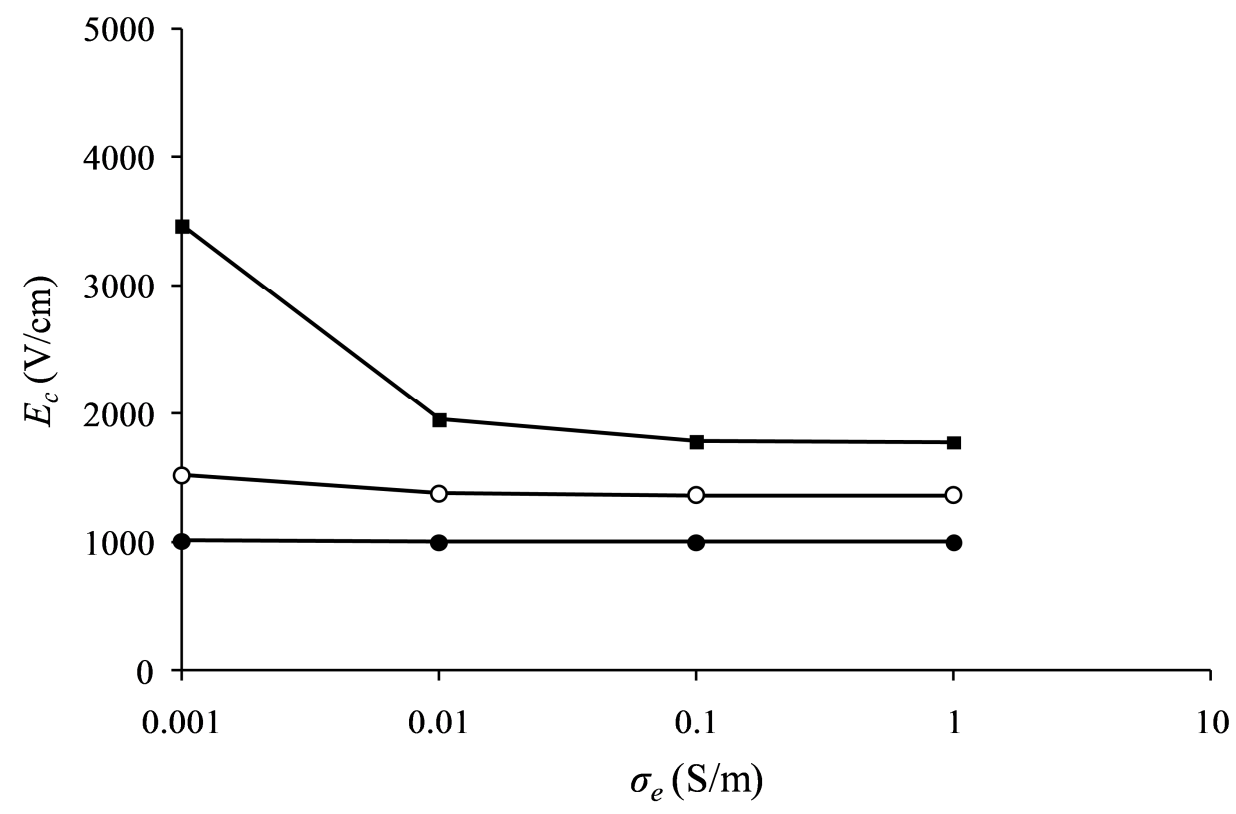

Fig. 9 Modeled electric field required to achieve three different permeabilization degrees (i.e. relative pores area) plotted against extracellular conductivity $\left(\sigma_{e}\right)$. The relative pores areas are: $1 \times 10^{-5}(\bullet), 3 \times 10^{-5}(\circ)$ and $1 \times 10^{-4}(\mathbf{\square})$.

\section{Conclusion}

Experimental data reported in the scientific literature shows that the amount of permeabilization depends on the conductivity of the extracellular medium. In particular, if the medium conductivity decreases then it is necessary to deliver a pulse of larger field amplitude in order to achieve the same amount of permeabilization. Here we demonstrate that such phenomenon can be explained as being the consequence of the dependence of membrane conductivity on induced transmembrane potential when pulses are applied.

Extracellular conductivities of about $1 \mathrm{~S} / \mathrm{m}$ and $0.1 \mathrm{~S} / \mathrm{m}$ yield very similar results in terms of permeabilization, however, for lower conductivities $(<0.01 \mathrm{~S} / \mathrm{m})$ our model predicts that significantly higher field magnitudes may be required to achieve the same amount of permeabilization

It is worth noting that a significant partial result found out in this study is that cell suspension conductance increase due to electroporation as a function of $E$ can be well reproduced by numerical simulations in which membrane conductivity dependence to $V_{m}$ is modeled with functions that impose an abrupt rise, such as exponential functions or power functions. 


\section{Appendix}

\subsection{Mathematical prediction of the direct influence of extracellular conductivity on the increase of transmembrane potential.}

In the case of a spherical cell in suspension it is possible to obtain an expression for the increase of transmembrane potential in response to an electric field pulse of magnitude $E^{26}$ :

$$
V_{m}=f_{s} E R \cos (\theta)\left[1-e^{\frac{-t}{\tau}}\right]
$$

where $f_{s}$ is a multiplying factor described in Eq. 10, $R$ is the cell radius, $\theta$ is the angle between the electric field direction and the radius at which $V_{m}$ is assessed, $t$ is the time since the beginning of the pulse and $\tau$ is a time constant described in Eq. 11.

$$
f_{s}=\frac{3 \sigma_{e}\left[3 d R^{2} \sigma_{i}+\left(3 d^{2} R-d^{3}\right)\left(\sigma_{m}-\sigma_{i}\right)\right]}{2 R^{3}\left(\sigma_{m}+2 \sigma_{e}\right)\left(\sigma_{m}+\frac{1}{2} \sigma_{i}\right)-2(R-d)^{3}\left(\sigma_{e}-\sigma_{m}\right)\left(\sigma_{i}-\sigma_{m}\right)},
$$

where $\sigma_{e}$ is the extracellular conductivity, $\sigma_{i}$ is the intracellular conductivity, $\sigma_{m}$ is the membrane conductivity and $d$ is the membrane thickness.

$$
\tau=\frac{R \frac{\varepsilon_{m}}{d}}{\frac{2 \sigma_{e} \sigma_{i}}{2 \sigma_{e}+\sigma_{i}}+\frac{R}{d} \sigma_{m}}
$$

where $\varepsilon_{m}$ is the membrane permittivity.

Taking into consideration realistic values for the geometry and for the dielectric parameters, it can be shown that these expressions indeed yield an effect of the extracellular conductivity $\left(\sigma_{e}\right)$ on the transmembrane potential: 1) as $\sigma_{e}$ decreases $f_{s}$ decreases and therefore the maximum level that $V_{m}$ can achieve also decreases and 2) as $\sigma_{e}$ decreases, the time constant $\tau$, increases and consequently, for a finite duration pulse, the membrane is exposed for a shorter period to the maximum voltage. However, as discussed in ${ }^{10}$, considering geometric and dielectric realistic values, the calculated $V_{m}$ dependence on $\sigma_{e}$ cannot match quantitatively what is observed experimentally. For instance, in the case of a decrease in extracellular conductivity from $0.1 \mathrm{~S} / \mathrm{m}$ to $0.01 \mathrm{~S} / \mathrm{m}$ the increase in $E_{c}$ according to $f_{s}$ would be below to $3 \%\left(\sigma_{m}=5 \times 10^{-7} \mathrm{~S} / \mathrm{m}, \sigma_{i}=0.5\right.$ $\mathrm{S} / \mathrm{m}, \mathrm{d}=5 \mathrm{~nm}, \mathrm{R}=5 \mu \mathrm{m})$ and the time constant would increase from $0.35 \mu \mathrm{s}$ to $2.53 \mu \mathrm{s}\left(\varepsilon_{m}=\right.$ $5.64 \times \varepsilon_{0}$ ), which is not very significant compared to the standard duration of electroporation pulses $(100 \mu \mathrm{s})$. Both figures are far from justifying the huge increase in $E_{c}$ that is observed in the curve A of Fig. 1, implying that another mechanism must exist in order to justify such experimental increase. More importantly, the above expressions for $f_{s}$ and $\tau$ do not predict the fact 
that $E_{c}$ sensitivity to $\sigma_{e}$ depends on the permeabilization level to be achieved, as illustrated in the cases B and D of Fig. 1.

\subsection{Dependence of critical field magnitude on extracellular conductivity when membrane conductivity is modeled with a sigmoid function.}

The curves depicted in Fig. 10 have been obtained with the following sigmoid model for $\sigma_{m}\left(V_{m}\right)$ :

$\sigma_{m}=\sigma_{m 0}+\sigma_{\text {pores }}\left(e^{-e^{-10\left(V_{m}-0.75\right)}}\right)$

This expression was selected because of having an abrupt increase in value around a threshold value (i.e. $0.75 \mathrm{~V}$ ). We did not even tried to adjust its values so that it produced a reasonable reproduction of the cell suspension conductance measurements as we did with the power function in the previous section (Fig. 6). As a matter of fact, we verified that such modeling by this sigmoid function was quite poor (data not reported here). Nonetheless, it can be observed that qualitatively the same behavior is obtained in terms of dependence of $E_{c}$ on $\sigma_{e}$ (Fig. 10 compared to Fig. 9).

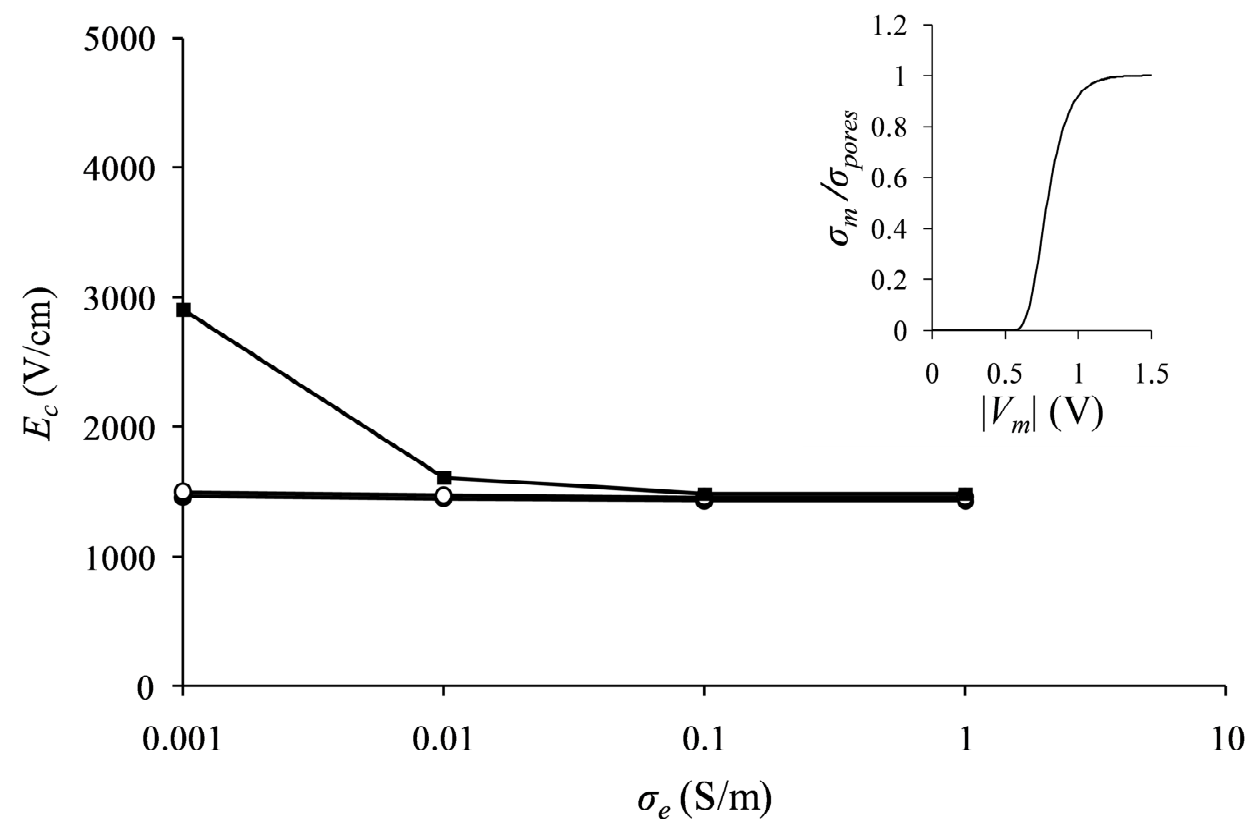

Fig. 10 Modeled electric field magnitude required to achieve three different permeabilization degrees when the membrane conductivity model consists of a sigmoid function (see insert) instead of an exponential function. $E_{c}$ is plotted against extracellular conductivity $\left(\sigma_{e}\right)$. The relative pores areas are: $1 \times 10^{-5}(\bullet), 3 \times 10^{-5}(\circ)$ and $1 \times 10^{-4}($ 


\section{Acknowledgements}

This work was supported by grants from: CNRS, Institute of cancerology Gustave-Roussy, University Paris Sud, INCa (National Institute of Cancer, France - contract number 07/3D1616/Doc-54-3/NG-NC) and French National Agency (ANR) through Nanoscience and Nanotechnology program (Nanopulsebiochip $n^{\circ}$ ANR-08-NANO-024).

AI's research is currently supported by a Ramón y Cajal fellowship from the Spanish Ministry for Science and Innovation. 


\section{References}

1 E. Neumann, Bioelectrochem. Bioenerget., 1992, 28, 247-267 (DOI:DOI: 10.1016/03024598(92)80017-B).

2 J. Teissie, N. Eynard, M. C. Vernhes, A. Benichou, V. Ganeva, B. Galutzov and P. A. Cabanes, Bioelectrochemistry, 2002, 55, 107-112.

3 T. Oshima and M. Sato, Adv. Biochem. Eng. Biotechnol., 2004, 90, 113-133.

4 M. J. Jaroszeski, R. Heller and R. Gilbert, in , ed. nonymous Humana Press, Totowa, New Jersey, 2000,

5 A. Gothelf, L. M. Mir and J. Gehl, Cancer Treat. Rev., 2003, 29, 371-387.

6 B. Rubinsky, G. Onik and P. Mikus, Technol. Cancer. Res. Treat., 2007, 6, 37-48.

7 S. Jayaram, G. S. P. Castle and A. Margaritis, Appl. Microbiol. Biotechnol., 1993, 40, 117-122.

8 V. L. Sukhorukov, H. Mussauer and U. Zimmermann, J. Membr. Biol., 1998, 163, 235-245.

9 C. S. Djuzenova, U. Zimmermann, H. Frank, V. L. Sukhorukov, E. Richter and G. Fuhr, Biochim. Biophys. Acta, 1996, 1284, 143-152.

10 G. Pucihar, T. Kotnik, M. Kanduser and D. Miklavcic, Bioelectrochemistry, 2001, 54, 107115.

11 E. Ferreira, E. Potier, D. Logeart-Avramoglou, S. Salomskaite-Davalgiene, L. M. Mir and H. Petite, Gene Ther., 2008, 15, 537-544 (DOI:10.1038/gt.2008.9).

12 Y. Antov, A. Barbul, H. Mantsur and R. Korenstein, Biophys. J., 2005, 88, 2206-2223 (DOI:10.1529/biophysj.104.051268).

13 M. Puc, S. Corovic, K. Flisar, M. Petkovsek, J. Nastran and D. Miklavcic, Bioelectrochemistry, 2004, 64, 113-124 (DOI:10.1016/j.bioelechem.2004.04.001).

14 M. P. Rols and J. Teissie, Biophys. J., 1998, 75, 1415-1423 (DOI:10.1016/S00063495(98)74060-3).

15 M. J. van den Hoff, W. T. Labruyere, A. F. Moorman and W. H. Lamers, Nucleic Acids Res., 1990, 18, 6464.

16 A. J. Sale and W. A. Hamilton, Biochim. Biophys. Acta, 1968, 163, 37-43.

17 F. Riemann, U. Zimmermann and G. Pilwat, Biochim. Biophys. Acta, 1975, 394, 449-462. 
18 J. Teissie and M. P. Rols, Biophys. J., 1993, 65, 409-413 (DOI:10.1016/S00063495(93)81052-X).

19 R. Stämpfli, An. Acad. Bras. Cienc, 1958, 30, 57-63.

20 U. Zimmermann, G. Pilwat and F. Riemann, Biophys. J., 1974, 14, 881-899

(DOI:10.1016/S0006-3495(74)85956-4).

21 K. Kinosita Jr, I. Ashikawa, N. Saita, H. Yoshimura, H. Itoh, K. Nagayama and A. Ikegami, Biophys. J., 1988, 53, 1015-1019 (DOI:10.1016/S0006-3495(88)83181-3).

22 I. G. Abidor, V. B. Arakelyan, L. V. Chernomordik, Y. A. Chizmadzhev, V. F. Pastushenko and M. P. Tarasevich, J Electroanal Chem, 1979, 104, 37-52 (DOI:DOI: 10.1016/S00220728(79)81006-2).

23 K. C. Melikov, V. A. Frolov, A. Shcherbakov, A. V. Samsonov, Y. A. Chizmadzhev and L. V. Chernomordik, Biophys. J., 2001, 80, 1829-1836 (DOI:10.1016/S0006-3495(01)76153-X).

24 R. W. Glaser, S. L. Leikin, L. V. Chernomordik, V. F. Pastushenko and A. I. Sokirko, Biochim. Biophys. Acta, 1988, 940, 275-287.

25 T. Kotnik, F. Bobanović and D. Miklavc`ičn, Bioelectrochem. Bioenerget., 1997, 43, 285-291 (DOI:DOI: 10.1016/S0302-4598(97)00023-8).

26 T. Kotnik, D. Miklavcic and T. Slivnik, Bioelectrochem. Bioenerget., 1998, 45, 3-16 (DOI:DOI: 10.1016/S0302-4598(97)00093-7).

27 R. Benz and U. Zimmermann, Biochim. Biophys. Acta, 1980, 597, 637-642.

28 K. Kinosita Jr and T. Y. Tsong, Biochim. Biophys. Acta, 1979, 554, 479-497.

29 I. Zudans, A. Agarwal, O. Orwar and S. G. Weber, Biophys. J., 2007, 92, 3696-3705 (DOI:10.1529/biophysj.106.097683).

30 J. Gimsa, T. Muller, T. Schnelle and G. Fuhr, Biophys. J., 1996, 71, 495-506 (DOI:10.1016/S0006-3495(96)79251-2).

31 W. Krassowska and P. D. Filev, Biophys. J., 2007, 92, 404-417

(DOI:10.1529/biophysj.106.094235).

32 T. R. Gowrishankar and J. C. Weaver, Proc. Natl. Acad. Sci. U. S. A., 2003, 100, 3203-3208 (DOI:10.1073/pnas.0636434100).

33 Z. Vasilkoski, A. T. Esser, T. R. Gowrishankar and J. C. Weaver, Phys. Rev. E. Stat. Nonlin Soft Matter Phys., 2006, 74, 021904. 
34 S. Koneshan, J. C. Rasaiah, R. M. Lynden-Bell and S. H. Lee, The Journal of Physical Chemistry B, 1998; 1998, 102, 4193-4204.

35 N. Pavselj, Z. Bregar, D. Cukjati, D. Batiuskaite, L. M. Mir and D. Miklavcic, IEEE Trans. Biomed. Eng., 2005, 52, 1373-1381 (DOI:10.1109/TBME.2005.851524).

36 P. Silley and S. Forsythe, J. Appl. Bacteriol., 1996, 80, 233-243.

37 G. R. Smith and M. S. Sansom, Biophys. Chem., 1999, 79, 129-151.

38 D. P. Tieleman, BMC Biochem., 2004, 5, 10 (DOI:10.1186/1471-2091-5-10).

39 M. Tarek, Biophys. J., 2005, 88, 4045-4053 (DOI:10.1529/biophysj.104.050617).

40 J. D. Hickey and R. Gilbert, DNA Cell Biol., 2003, 22, 823-828

(DOI:10.1089/104454903322625037).

41 S. Takashima, Biophys. J., 1979, 26, 133-142 (DOI:10.1016/S0006-3495(79)85240-6).

42 A. Ivorra and B. Rubinsky, Bioelectrochemistry, 2007, 70, 287-295

(DOI:10.1016/j.bioelechem.2006.10.005).

43 S. I. Sukharev, V. A. Klenchin, S. M. Serov, L. V. Chernomordik and Y. Chizmadzhev, Biophys. J., 1992, 63, 1320-1327 (DOI:10.1016/S0006-3495(92)81709-5).

44 M. Golzio, J. Teissie and M. P. Rols, Proc. Natl. Acad. Sci. U. S. A., 2002, 99, 1292-1297 (DOI:10.1073/pnas.022646499).

45 S. Satkauskas, M. F. Bureau, M. Puc, A. Mahfoudi, D. Scherman, D. Miklavcic and L. M. Mir, Mol. Ther., 2002, 5, 133-140 (DOI:10.1006/mthe.2002.0526). 